\title{
The influence of rates of drying and wetting on measurements of soil water retention curves
}

\author{
Arash Azizi ${ }^{*}$, Ashutosh Kumar $^{1}$, Mwajuma Ibrahim Lingwanda ${ }^{1}$ and David Geoffrey Toll ${ }^{1}$ \\ ${ }^{1}$ Department of Engineering, Durham University, UK
}

\begin{abstract}
The water retention curve is fundamental for a comprehensive description of the hydro-mechanical behaviour of unsaturated soils. The water retention testing system developed at Durham University allows direct and continuous measurement of suction using a high capacity tensiometer, water content determined from mass readings of a digital balance and measurements of volume change. The system was modified to accommodate an additional tensiometer to measure suction at the top besides the existing one at the bottom of the soil specimen. Soil specimens were subjected to drying and wetting following two procedures: discrete measurements carried out in stages to ensure equalisation and continuous measurement at different rates. All suctions measured during continuous and discrete measurements were very close at high saturation degrees. At lower saturation degrees, the suction values from the top and bottom of the specimen deviated from suctions observed in discrete measurements. This deviation in suction values was more evident in accelerated drying and wetting patterns. This can be explained by the fact that water permeability reduces with the decrease in saturation levels.
\end{abstract}

\section{Introduction}

The relationship between the amount of water stored in soil pores and suction, known as Soil Water Retention Curve (SWRC), is essential to understand the hydromechanical behaviour of soils under unsaturated conditions. It has been commonly used to estimate various properties of unsaturated soils such as shear strength and permeability $[1,2]$. The dependency of the SWRC on soil density and its hysteretic nature observed along drying and wetting paths have been of great interest in the field of laboratory testing of unsaturated soils [3, 4].

Many devices have been developed to measure the water retention behaviour of soils at laboratory scales. A key consideration in the laboratory determination of the SWRC is how to control or measure soil suction. The most common suction-controlled techniques used for water retention testing are axis translation, vapour equilibrium and osmotic suction [5, 6]. All these techniques suffer from the long testing time that is required to ensure water equalisation is achieved across soil specimens upon imposed suction levels. Following the development of advanced suction probes, several attempts have been made to measure rather than control suction for water retention testing, thereby using them as an alternative technique to determine the SWRC [7-9]. The water retention testing system developed at Durham University allows measurements of volume change of a soil specimen and uses a high capacity tensiometer and a digital balance to measure soil suction and water content $[10,11]$. The high capacity tensiometer provides rapid and direct measurements of suction while the soil specimen is subjected to water evaporation or continuous wetting through water droplets, thereby allowing the SWRC to be obtained much faster and more representative of the natural mechanism (true drying) than other standard methods. However, the accuracy of measurements during continuous drying and wetting and the state of equilibrium of water and suction within the soil specimen have not been fully addressed and need further investigations.

In this paper, the current version of the water retention system developed at Durham University is described. The device has been recently modified to accommodate an additional tensiometer to measure suction levels at the top besides the existing one that measures suction at the bottom of the soil specimen. This allows the suction gradient generated along the height of the specimen to be detected. The wetting capability of the system has also been improved to allow sprinkling of water at multiple locations on the specimen surface. The modified water retention system is currently employed by the "Transport Africa" project where the water retention behaviours of naturally occurring soils, consisting different percentages of fine fractions, from subgrades of roads and railway embankments are being studied under cycles of drying and wetting. The research presented in this paper aims at studying the significance of lack of suction equalisation and the effect of rates of drying and wetting on the measurements of the water retention curves of a subgrade soil subjected to continuous drying and wetting.

Corresponding author: arash.azizi@ durham.ac.uk 


\section{Water retention testing system}

Figure 1 shows a sketch of the water retention device used in this study. The device allows continuous measurements of suction, water content and volume changes of a soil specimen subjected to cycles of drying and wetting. The specimen is placed on the plate of a cube shaped frame while enclosed in a shroud. The suction is measured using high capacity tensiometers capable of measuring suction up to $2 \mathrm{MPa}[8,12]$, which satisfies most of the suction range that is encountered in many practical geotechnical problems. Two tensiometers are mounted in the centre, one at the top and the other at the bottom of the specimen, while supported carefully to ensure decent contacts between the soil and the sensing face of the tensiometers. The area covered by the tensiometer placed on the top is $3.14 \mathrm{~cm}^{2}$ which is very small compared to the area of the top surface of the specimen $\left(78.5 \mathrm{~cm}^{2}\right)$. The frame accommodates six displacement transducers to measure axial and radial deformations, thereby monitoring volume changes of the specimen. All instruments are connected to a data logger that sits inside the frame and communicates with a realtime data acquisition system remotely. The frame housing the specimen along with all instruments are placed on a precise digital balance which allows determining water content changes of the specimen from mass readings. The cables connecting different instruments to the data logger are supported cautiously to minimize the influence of stiffness or movement of the cables on the mass measurements. To improve the wetting part of the system, a stand is placed adjacent to the frame which holds water pipes and allows sprinkling of water droplets onto the top surface of the specimen at a controlled rate.

Drying paths are applied while the soil specimen is exposed to the atmosphere. The water evaporates from the top and lateral surfaces of the specimen while the evaporation rate can be controlled by adjusting the exposure of the holes punched in the shroud. Wetting paths are imposed by sprinkling water on the top surface of the specimen at controlled rates regulated by solenoid valves. The current setup of the wetting system consists of four nozzles that allow water drops to be injected at four locations on the top surface of the specimen, each having a distance of about $25 \mathrm{~mm}$ from the centre.

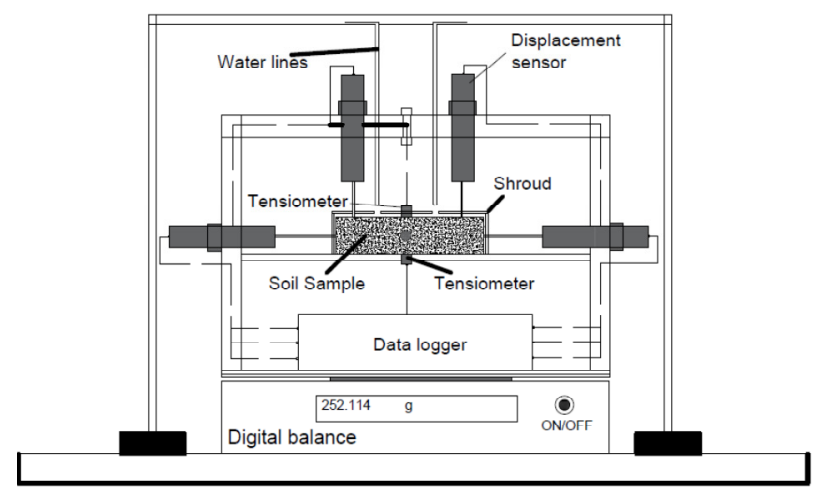

Fig. 1. Water retention system developed at Durham University (Modified after Toll et al. 2015).

\section{Testing procedure}

The soil used in this study was taken from the subgrade of a low volume road constructed in the North East of Tanzania. It is a clayey silt consisting of $43 \%$ clay, $44 \%$ silt and $15 \%$ sand. The plasticity index and specific gravity of the soil are $16.5 \%$ and 2.66 , respectively. The oven dried powdered soil was mixed with water and kept for at least 24 hours in a closed plastic bag to ensure water equalisation. The cylindrical specimen, having a diameter of $100 \mathrm{~mm}$ and a height of $25 \mathrm{~mm}$, was then statically compacted at a dry density of $1350 \mathrm{Mg} / \mathrm{m}^{3}$ and moisture content of $35 \%$ i.e. just wet of optimum $\left(S_{r}>\right.$ $95 \%)$. The ratio between the surface area exposed to the atmosphere and the volume of the specimen was equal to $0.075 \mathrm{~mm}^{-1}$. [12] reported that no significant water gradient across the soil specimens prepared with similar ratios was observed during drying. To maintain constant evaporation rates during testing, all measurements were conducted in a temperature-controlled room with temperature $(T)$ of $21 \pm 0.5^{\circ} \mathrm{C}$ and relative humidity $(R H)$ of $34 \%$.

The water retention tests were carried out while the soil specimens were subjected to drying and wetting following two procedures: 1) discrete measurements carried out in stages to ensure equalisation, the drying and wetting tests with discrete measurements are titled as "DS" and "WS", respectively; 2) continuous measurements with drying and wetting with no pauses, carried out at different rates to study the effect of the imposed rates on the measurement of the water retention curves of the tested soil. For discrete measurements, the drying path was carried out by keeping the specimen exposed to atmosphere in a temperature and relative humidity controlled room whereas the wetting path was imposed in a relative humidity controlled chamber $(R H \geq$ $95 \%)$. The hydraulic boundary conditions were kept similar to the continuous measurements where the specimen was exposed to the atmosphere from the top and lateral surfaces. The drying and wetting tests with continuous measurements are titled as "DC" and "WC", respectively. The rate of drying was accelerated from DC01 to DC03 and the rates of wetting from WC01 to WC03. The description of the tests performed is presented in Table 1 . The rate of drying and wetting is shown in terms of $\Delta w / h$ where $\Delta w$ is changes in the gravimetric water content and $h$ is the elapsed time (hours).

Table 1. The description of the tests carried out.

\begin{tabular}{|c|c|c|c|}
\hline Title & Hydraulic path & Continuity & Rate $(\% \Delta w / h)$ \\
\hline DS & Drying & Staged & -0.04 (average) \\
\hline DC01 & Drying & Continuous & -0.10 \\
\hline DC02 & Drying & Continuous & -0.13 \\
\hline DC03 & Drying & Continuous & -0.21 \\
\hline WS & Wetting & Staged & 0.025 (average) \\
\hline WC01 & Wetting & Continuous & 0.05 \\
\hline WC02 & Wetting & Continuous & 0.07 \\
\hline WC03 & Wetting & Continuous & 0.10 \\
\hline
\end{tabular}


Figure 2 shows changes in suction $(s)$ and gravimetric water content $(w)$ with the elapsed time for the two procedures: the continuous drying test (DC01) followed by the continuous wetting test (WC01), and the staged drying test (DS) followed by the staged wetting test (WS). DS and WS were carried out by halting the tests at different periods, wrapping the specimens in plastic bags and keeping in a $R H$ controlled chamber for at least $24 h$. The suction measured after the equalisation periods was constant so ensuring equilibrium was achieved and the data points (DS and WS) present suction and water content at hydraulic equilibrium. The specimens from DC01 and DS were dried to the water content of $29.1 \%$ ( $s=986 \mathrm{kPa}$ for DC01 and $s=1073$ $\mathrm{kPa}$ for DS). The specimens were then wetted until suction reached $15 \mathrm{kPa}(w=33.7 \%)$ for $\mathrm{WC} 01$ and 43 $\mathrm{kPa}(w=31.8 \%)$ for WC. The duration of the drying and wetting tests with the discrete measurements (DS-WS) was about two times longer than the slowest tests with the continuous measurements (DC01-WC01). It has to be pointed out that the two procedures used hereby measure SWRCs considerably quicker than the other standard techniques that control suction during water retention testing.

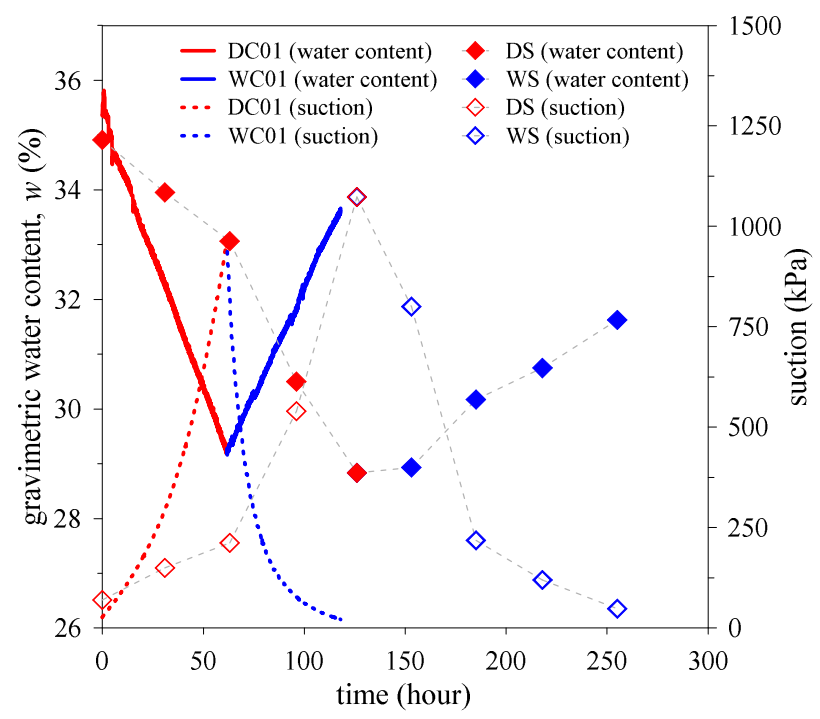

Fig. 2. Measurements of suction and gravimetric water content for DC01 followed by WC01 and for DS followed by WS.

Figure 3 shows the variations of the gravimetric water content $(\Delta w)$ with the elapsed time for the tests with continuous measurements. The fairly straight lines imply the water evaporation and sprinkling took place at constant rates along the entire drying and wetting paths. The drying tests were performed at three different rates. The fastest drying test, DC03, was performed within $25 \mathrm{~h}$ $(\Delta w=-0.21 \% / h)$ which was about two times faster than the slowest drying test DC01 $(\Delta w=-0.10 \% / h)$. The wetting tests were also performed at three different rates. Since water was sprinkled only on four locations on the top of the specimen, hydraulic equalisation for wetting was expected to take longer than the drying paths in which the water could evaporate uniformly from the top and lateral surfaces. Hence, the wetting tests were performed at rates lower than the rates of drying tests. The fastest and slowest wetting tests, WC03 and WC01, were performed at the rate of $\Delta w=0.10 \% / h$ and $0.05 \% / h$, respectively.

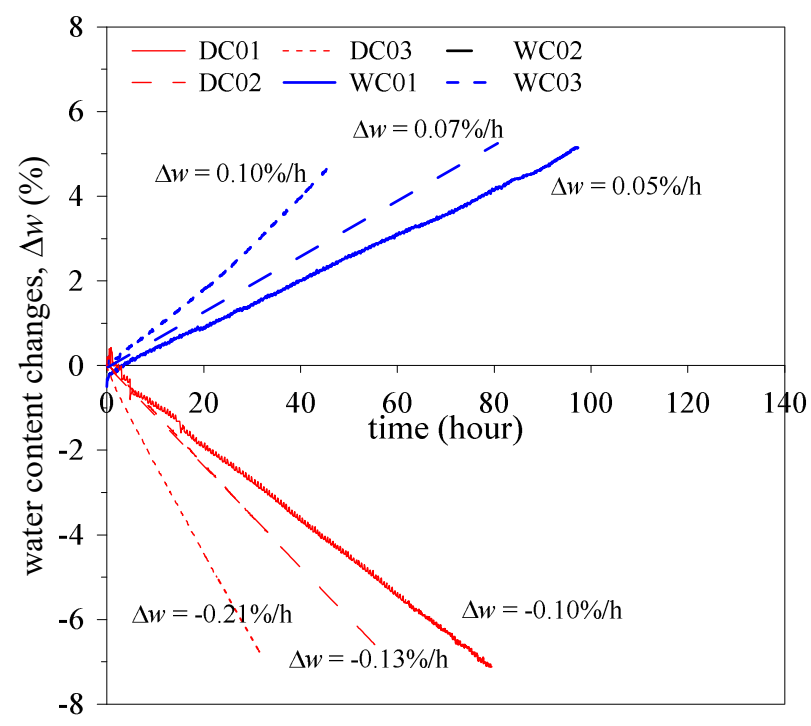

Fig. 3. Variation of water content for tests with continuous measurements performed at different drying and wetting rates.

\section{Results and discussion}

The discrete measurements carried out in stages (DS and WS) represent suction and water content values at hydraulic equilibrium, however, the state of equilibrium for the SWRCs obtained from the continuous measurements needs to be assessed due to continuous variations of water content. In the following section, the SWRCs obtained from continuous drying and wetting tests are compared with the SWRCs obtained at equilibrium (from discrete measurements) and the effect of the rate of drying and wetting on the measurements is discussed.

\subsection{Rate effect on drying water retention curves}

Figure 4 compares the drying SWRCs obtained from DC01 to DC03 with the drying SWRC obtained from DS in terms of volumetric water content $\left(\theta_{v w}\right)$ and suction $(s)$. The SWRCs from continuous drying are plotted against two suction values measured at the top and bottom of the specimens. The water retention model proposed by [13] (VG model) was used to simulate the soil water retention data points obtained from the discrete measurements (DS):

$$
\theta_{v w}=\theta_{s} \frac{1}{\left[1+(\alpha s)^{n}\right]^{m}}
$$

where $\theta_{s}=48$ is saturated volumetric water content; $\alpha=$ $0.007, n=1.08$, and $m=1-1 / n$ are model parameters. The air entry value was found to be about $140 \mathrm{kPa}$. Figure 4 shows also the curve predicted by the model 
which fits the data points well. The predicted curve facilitates the comparison between the SWRC from DS (at equilibrium) and the SWRCs obtained from the continuous measurements.

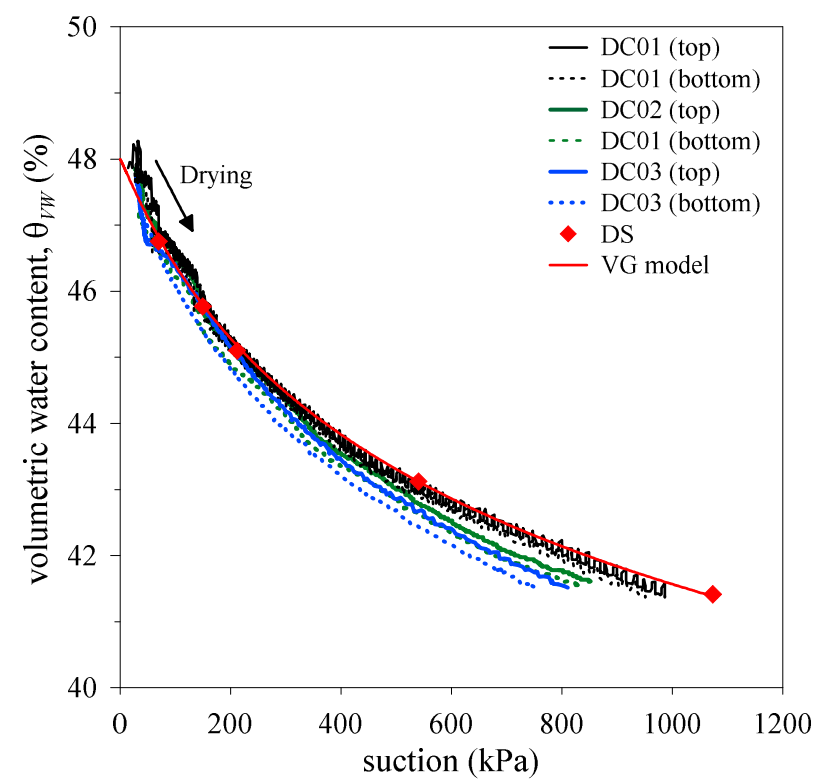

Fig. 4. The drying soil water retention curves obtained by continuous and discrete measurements.

Since all specimens were prepared at a very high saturation degree, the suction values measured at the beginning of the drying tests were low $(s \leq 10 \mathrm{kPa})$. All water retention curves are in good agreement at suction levels lower than $200 \mathrm{kPa}\left(\theta_{v w}>45 \%\right)$, with the exception of the SWRCs of DC03. The drying curves of DC01 to DC03 deviated from the one of DS as suction levels exceeded $200 \mathrm{kPa}$. This deviation is more evident in the tests carried out at the accelerated drying patterns. The SWRCs of DC02 and DC03 underestimated the suctions at equilibrium.

The drying SWRCs obtained from the suction measured at the top were found to be above those obtained from the suction measured at the bottom of the specimen as shown in Figure 4. This implies that the suction from the top was greater than the one from the bottom of the specimens during drying. This can be explained as the pore water evaporated with faster drying from the top surface with respect to the core of the specimen. However, the two drying SWRCs of DC01 carried out at the decelerated evaporation rate were found to be very close.

Figure 5 shows the difference in suctions $(\Delta s)$ measured at the bottom $s_{\text {bottom }}$ and top $s_{\text {top }}$ of the specimens. $\Delta s$ was negligible at the beginning of the tests, which confirms the specimens were at hydraulic equilibrium. $\Delta s$ increased as drying progressed and intensified with the increase in the drying rate. The increase in the rate accelerated evaporation from the top surface, and in turn, increased suction gradient along the height of the specimens from DC01 to DC03. The gradual increase in the suction gradient with drying can be justified by the fact that the water permeability of the tested soil decreased with the increase in suction. Hence, the water distribution within the specimen decelerated with desaturation.

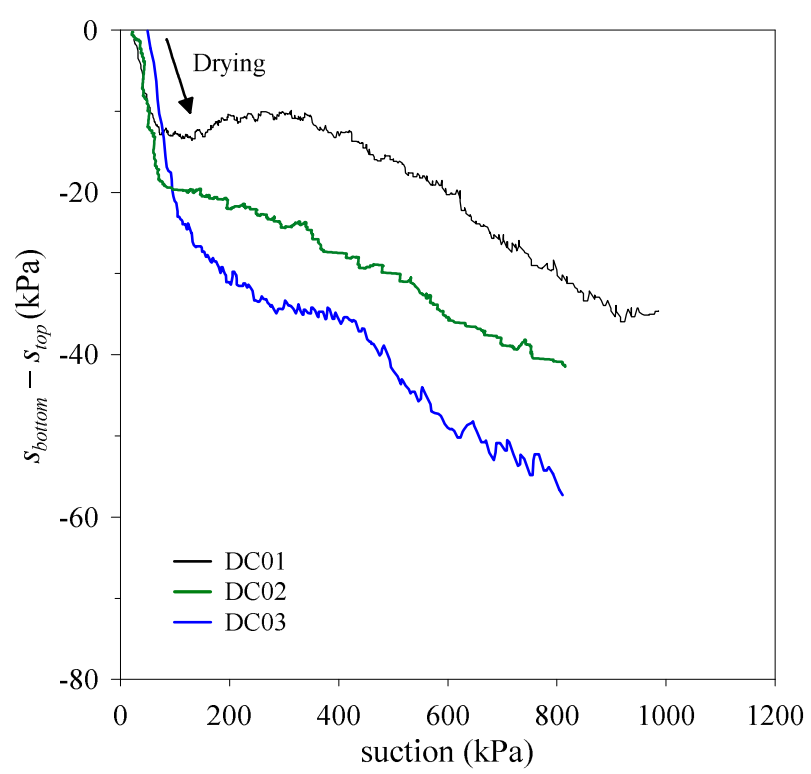

Fig. 5. Difference in suctions $\Delta s$ measured at the top and bottom of the specimens from DC01 to DC03.

At the end of the drying paths, $\Delta s$ from DC03 was $-55 \mathrm{kPa}$, which was about 2 times greater than the one of DC01. A similar sort of suction gradient is also generated along the radius of the specimen due to evaporation from the lateral surfaces.

The water permeability of unsaturated soils can be estimated by using the water permeability of saturated soils weighted by the relative permeability $k_{r}$. The relative permeability is dependent on degree of saturation $S_{r}$ and can be obtained from the soil water retention curve [14]:

$$
k_{r}=S_{r}{ }_{r}\left[\frac{\int_{0}^{s_{r}} d S_{r} / s}{\int_{0}^{1} d S_{r} / s}\right]^{2}
$$

Figure 6 shows the variation of the relative permeability with the degree of saturation for the tested soil, which was obtained from equation (2) using the drying SWRC predicted by the VG model. The difference between the suction obtained at equilibrium $s_{\text {discrete }}$ and from continuous measurements $s_{\text {continuous }}$ is also plotted in Figure 6. The difference between these two suction levels increased with desaturation and the rate of drying. It can be observed that $k_{r}$, and in turn the water permeability, decreases significantly as the saturation level decreases. This can delay the distribution of water across the specimen and suction equalisation during continuous drying. As a result, the SWRCs obtained from continuous drying differed from the SWRC measured at equilibrium as the degree of saturation and permeability decreased. This deviation 
intensified with the rate of drying due to faster evaporation from the specimen surface.

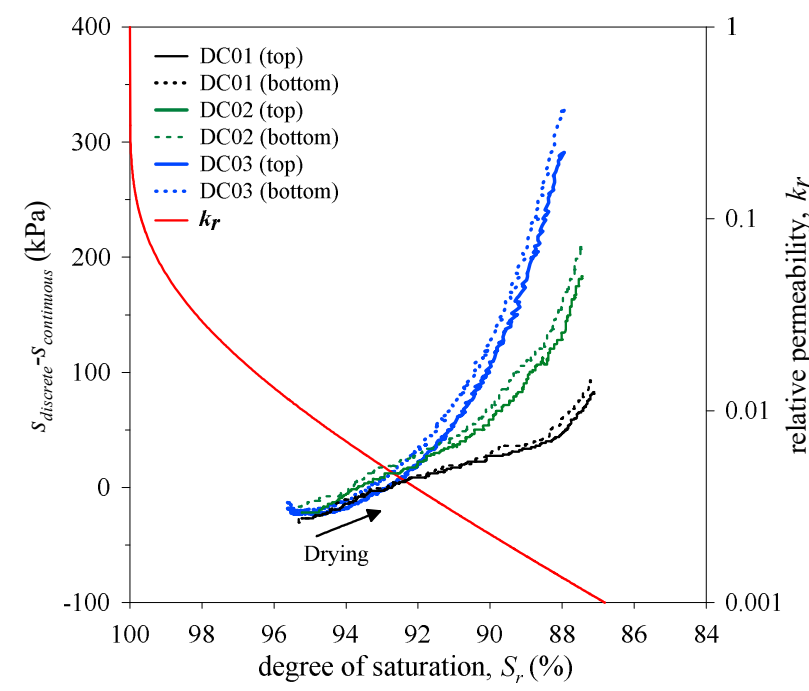

Fig. 6. Variation of the relative permeability and $s_{\text {discrete }}$ $s_{\text {continuous }}$ with degree of saturation.

The results showed that decelerating the drying rate reduced the suction gradient, hence resulted in obtaining the SWRC (DC01) very close to the one measured at equilibrium. It should be noted that the soil used in this study has significant clay content $(42 \%)$ and silt content $(43 \%)$. This deviation observed in the drying SWRCs introduced by hydraulic gradients across the specimen might be less significant in materials with less fine fractions and higher permeability as suggested by [12].

\subsection{Rate effect on wetting water retention curves}

Figure 7 shows the wetting SWRCs obtained from continuous measurements (WC01 to WC03) and from discrete measurements (WS). At the beginning of the wetting paths, the suction values from the top and bottom of the specimen were almost the same, which implies that the specimens were at hydraulic equilibrium. The wetting SWRC of WS was reproduced using the VG model where $\theta_{s}=47, \alpha=0.017, n=1.72$. At the initial stage of wetting, the increase in the volumetric water content of the specimens is associated with the delayed suction response since the two tensiometers provide local measurements of suction in the centre of the specimens. All wetting curves obtained from continuous wetting lay above the SWRC obtained from discrete measurements. However, the wetting curves of WC01 were relatively close to the one of WS. The SWRCs of WC01 and WS merged as the volumetric water content increased to the values greater than $43 \%$. When the specimen was wetted, different suctions were measured at the top and bottom of the specimens. The water was sprinkled on the top surface of the specimen so suction from the top decreased faster than the one from the bottom of the specimen. Therefore, the SWRC obtained from the suction measured at the top lay below the SWRC from the suction measured at the bottom of the specimen as shown in Figure 7. The difference between the two curves of WC01 carried out at the decelerated wetting rate was less evident.

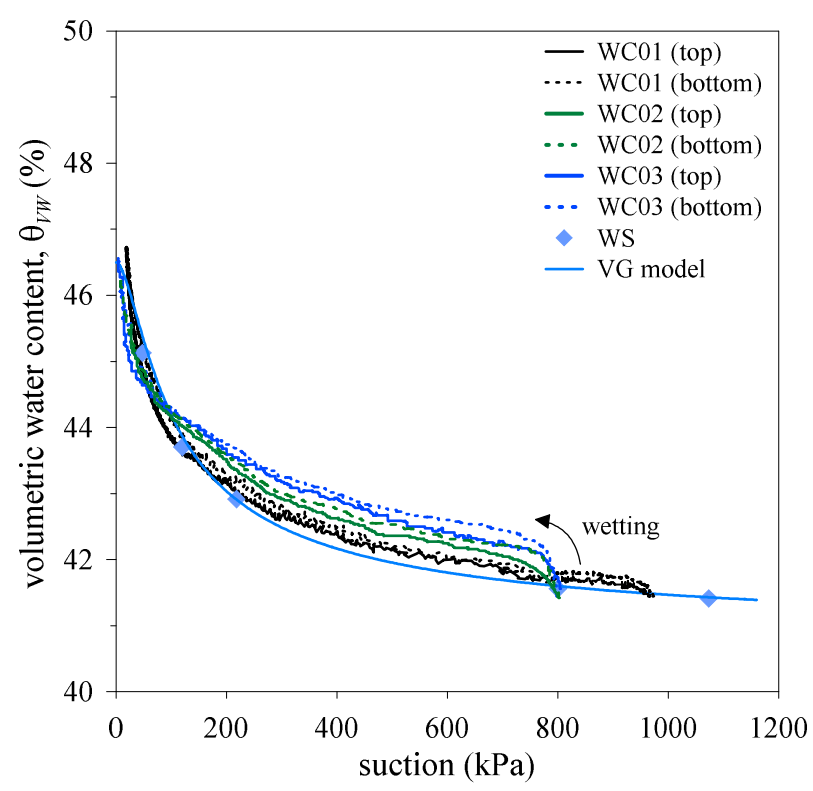

Fig. 7. The wetting soil water retention curves obtained by continuous and discrete measurements.

Figure 8 shows the difference in suction values measured at the top and bottom of the specimen $(\Delta s)$ for $\mathrm{WC} 01$ to $\mathrm{WC0} 3$ during the wetting process. At the beginning of the wetting paths, $\Delta s$ is negligible as the specimens were at hydraulic equilibrium. Once the wetting stage was started, the suction values differed immediately which was more evident as the wetting rate increased. The increase in the rate of wetting and the low permeability of the tested soil at high suction levels (lower saturation degrees) caused faster saturation of the top surface than the core and a greater suction gradient along the height of the specimen. As shown in Figure 8, $\Delta s$ dissipated gradually with the decrease in the suction levels, owing to the increase in the water permeability and faster distribution of water across the specimen as the wetting stage progressed and the degree of saturation increased.

Figure 9 shows the difference between suctions obtained from discrete and continuous measurements $s_{\text {discrete }}-s_{\text {continuous }}$ and the relative permeability with the degree of saturation. The difference between the two suction values was greater at lower saturation degrees. This can be explained by the low value of permeability $k_{r}$ at high suction levels. The distribution of water decelerated due to the low permeability; hence a greater suction gradient was generated across the specimen during continuous wetting. At the accelerated wetting patterns, the suction gradient increased, therefore, the SWRCs of WC02 and WC03 differed from the one of WS and distinctly overestimated the suction at equilibrium. However, the SWRC of WC01 carried out at the decelerated rate of wetting closely resembled the SWRC obtained from the discrete measurements. $k_{r}$ (and in turn the water permeability) increased as wetting progressed, thereby leading to faster distribution of water 
and dissipation of suction gradient across the specimen. The difference between the wetting SWRCs obtained from continuous and discrete measurements reduced when saturation degrees and water permeability increased.

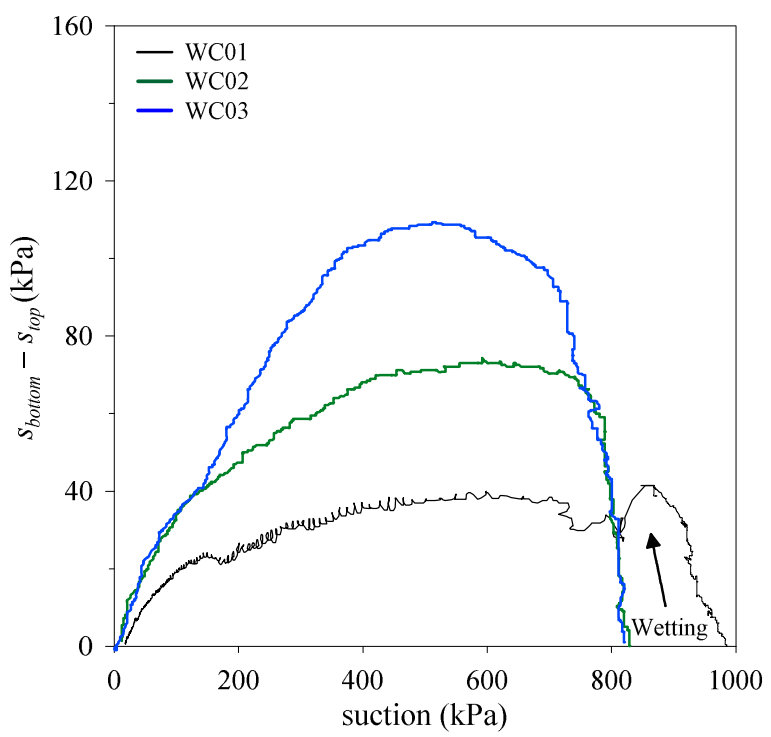

Fig. 8. Difference in suctions $\Delta s$ measured at the top and bottom of the specimens from WC01 to WC03.

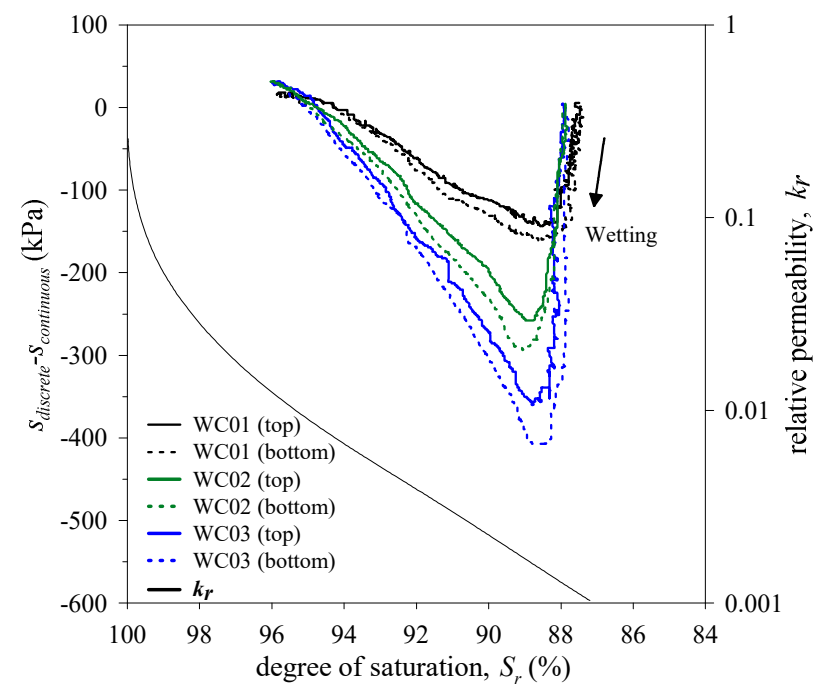

Fig. 9. Variation of the relative permeability and $s_{\text {discrete }}$ $s_{\text {continuous }}$ with degree of saturation.

Data presented hereby suggests that sprinkling water on the top surface of the specimen at the decelerated wetting rate can help to obtain the wetting water retention curves close to the curves obtained at the equilibrium condition. Further improvements can be made by increasing the number of nozzles to sprinkle water over the top surface of the specimen in more homogenised wetting patterns.

\section{Conclusion}

This paper describes the water retention testing system developed at Durham University which has been modified to allow suction measurements on the top in addition to the bottom of the specimen to measure suction gradient along the height, and sprinkling water at multiple locations on the specimen surface. Water retention tests were carried out at different rates of drying and wetting to study the effect of the imposed rates on the measurement of the SWRCs. The SWRCs obtained were compared to the SWRCs obtained from staged drying and wetting in which suction was measured at hydraulic equilibrium.

The results showed that the rates of drying and wetting can influence the measurements of SWRCs of the tested soil at lower degree of saturations. The suction gradient across the specimen was also found to be dependent on the degree of saturation and the imposed rates. The water permeability of the tested soils decreased significantly with the decrease in the degree of saturation. This delayed the distribution of the water, leading to a greater suction gradient across the specimen. Consequently, the SWRCs from continuous drying and wetting at lower saturation degrees deviated from the SWRC obtained at equilibrium. At accelerated drying and wetting patterns, the suction gradient was increased so this deviation was more evident. The SWRCs obtained from continuous drying and wetting carried out at decelerated rates closely resemble the SWRC obtained by the discrete measurements indicating that the accuracy of the measurements can be improved and suction values very close to the suctions at equilibrium can be measured.

\section{References}

1. D. G. Fredlund, A. Xing, S. Huang, Canadian Geotechnical Journal 31, (4), 553-546 (1994)

2. S. K. Vanapalli, D. G. Fredlund, D. E Pufahl, A. W. Clifton, Can. Geotech. J. 33, 379-392 (1996)

3. D. Gallipoli, S. J. Wheeler, M. Karstunen, Géotechnique 53, 1, 105-12 (2003)

4. A. Azizi, C. Jommi, G. Musso, Computer and Geotechnics 87, 86-98 (2017)

5. A. W. Bishop, I.B Donald, Proc. 5th Conf. On Soil Mechanics and Found Eng. 1, 13-21 (1961)

6. Y. J. Cui, P. Delage, Géotechnique 46, 2, 291-311 (1996)

7. P. F. Teixeira, F. A. M. Marinho, COBRASEG 1, 423-427 (2006)

8. D. G .Toll, S.D.N. Lourenço, J. Mendes, Engineering Geology, 165, 29-37 (2013)

9. R. Cardoso, G. Sarapajevaite, O. Korsun, S. Cardoso, L. Ilharco, Canadian Geotechnical Journal 54,1176-1183 (2017)

10. D. G. Toll, et al. Proceedings of the 6th AsiaPacific Conference on Unsaturated Soils. 15-22 (2015) 
11. G. Liu, D. Toll, L. Kong, J. Asquith, Geotechnical Testing Journal (2019)

12. S. D. N. Lourenço, D. Gallipoli, D. G. Toll, C. E. Augarde, F. Evans, Canadian Geotechnical Journal 48, 2, 327-335 (2011)

13. M. T. van Genuchten, Soil Sci Soc Am J. 44, 5, 8928 (1980)

14. Y. Mualem, Water Resources Research 12, 3, 513522 (1976) 